\title{
MULTIPLE EXISTENCE AND LINEAR STABILITY OF EQUILIBRIUM BALLS IN A NONLINEAR FREE BOUNDARY PROBLEM
}

\author{
BY
}

\author{
M. TANIGUCHI
}

Tokyo Institute of Technology, Japan

\begin{abstract}
This paper studies construction and linear stability of spherical interfaces in an equilibrium state in a two-phase boundary problem arising in activator-inhibitor models in chemistry. By studying the linearized eigenvalue problem near a given equilibrium ball, we show that the eigenvalues with nonnegative real parts are all real, and that they are characterized as values of a strictly convex function for specific discrete values of its argument. The stability is determined by the location of the zero points of this convex function. Using this fact, we present a criterion of stability in a useful form. We show examples and illustrate that stable equilibrium balls and unstable ones coexist near saddle-node bifurcation points in the bifurcation diagram, and a given equilibrium ball located far from bifurcation points is unstable and the eigenfunction associated with the largest eigenvalue consists of spherically harmonic functions of high degrees.
\end{abstract}

1. Introduction. This paper is concerned with a simultaneous equation of a free phase boundary $\Gamma(t)$ and a scalar function $v(x, t)$ in time $t>0$ and space $x \in \mathbf{R}^{N}$ $(N=2$ or 3$)$. We consider

$$
\begin{aligned}
\tau V_{\Gamma(t)} & =W(v)-(N-1) \alpha H \quad \text { on } \Gamma(t), t>0 \\
v_{t}-\Delta v & =G^{+}(v) \chi_{\Omega^{+}(t)}+G^{-}(v) \chi_{\Omega^{-}(t)} \quad \text { in } \mathbf{R}^{N}, t>0,
\end{aligned}
$$

with initial conditions

$$
\begin{aligned}
\Gamma(0) & =\Gamma_{0}, \\
v(x, 0) & =v_{0}(x) \quad \text { for } x \in \mathbf{R}^{N} .
\end{aligned}
$$

Here $\Gamma(t)$ is an embedded surface in $\mathbf{R}^{N}$, and it separates $\mathbf{R}^{N}$ into two disjoint regions $\Omega^{+}(t)$ and $\Omega^{-}(t) . \quad \Gamma(t)$ is a boundary of two different phases and is often called an interface. For every fixed $t>0$, let $\nu$ be the outward unit normal vector of $\Gamma(t)$ pointing from $\Omega^{+}(t)$ to $\Omega^{-}(t)$, and let $H$ be the mean curvature at each point of $\Gamma(t)$, that is, $H=(N-1)^{-1} \operatorname{div} \nu$. We denote by $V_{\Gamma(t)}$ the normal velocity of $\Gamma(t)$ pointing from $\Omega^{+}(t)$ 
to $\Omega^{-}(t)$. In the first equation, $\tau, \alpha$ are given positive numbers, and $\chi_{A}$ signifies the characteristic function of a set $A \subset \mathbf{R}^{N}$.

Our standing assumptions are as follows.

(A1) $G^{ \pm}(\cdot)$ is a real-valued function of class $C^{1}$ on a given interval $[\underline{v}, \bar{v}]$, respectively. Here $-\infty<\underline{v}<\bar{v}<+\infty$. The following conditions hold: $G^{+}(\bar{v})=0, G^{-}(\underline{v})=0$ and $G_{v}^{ \pm}(v)<0$ on $[\underline{v}, \bar{v}]$, respectively.

(A2) $W(\cdot)$ belongs to $C^{1}[\underline{v}, \bar{v}]$ with $W^{\prime}(v)<0$ for all $v \in(\underline{v}, \bar{v})$.

(A3) Inequality $\tau>\tau_{*}$ holds, where $\tau_{*}$ is a positive constant given by (3.1) in Sec. 3 .

This free boundary problem arises in activator-inhibitor (or propagator-controller) models that appear in chemistry or mathematical biology. See [5] and [11] for instance. Equation (1.1)-(1.2) is closely related to a reaction-diffusion equation of bistable type that describes these models. Let $u(x, t)$ and $w(x, t)$ be two chemical substances, an activator and an inhibitor. Then it is written as

$$
\varepsilon \tau_{0} u_{t}-\varepsilon^{2} \Delta u=u-u^{3}-w, \quad w_{t}-\Delta w=g(u, w)
$$

in $\mathbf{R}^{N}, t>0$. Here $g(u, w)$ is some function that is monotone decreasing in $w$ and monotone increasing in $u . \tau_{0}$ is a positive constant, and $\varepsilon$ is very small. The smallness of $\varepsilon$ leads to the generation and propagation of a transition layer where $u$ has a jump. From (1.3), the following equation

$$
\begin{aligned}
\tau_{0} V_{\Gamma(t)} & =W_{0}(w)-(N-1) \varepsilon H \quad \text { on } \Gamma(t), t>0, \\
w_{t}-\Delta w & =G^{+}(w) \chi_{\Omega^{+}(t)}+G^{-}(w) \chi_{\Omega^{-}(t)} \quad \text { in } \mathbf{R}^{N}, t>0,
\end{aligned}
$$

is derived as the asymptotic limit of $\varepsilon \rightarrow+0$. Here $W_{0}(\cdot)$ and $G^{ \pm}(\cdot)$ are some monotone decreasing functions. See Fife [5], Nishiura and Mimura [10], X-Y. Chen [3], and $\mathrm{X}$. Chen [2] for the derivation and the well-posedness of (1.4)-(1.5). Equation (1.4)-(1.5) is an example of (1.1)-(1.2) if $\alpha=\varepsilon$ and $\tau=\tau_{0}$.

A first step to study (1.4)-(1.5) is to investigate the existence and linear stability of radially symmetric equilibrium solutions. Ohta, Mimura, and Kobayashi [11] studied them for some special cases. X. Chen and the author [4] studied them for general $G^{ \pm}(\cdot)$, and proved the instability of those solutions when $\varepsilon>0$ is small enough.

This paper is an extension work of [4], and is devoted to the existence and linear stability of radially symmetric equilibrium solutions of (1.1)-(1.2) for every $\alpha>0$ including large $\alpha$ and for all $W(\cdot), G^{ \pm}(\cdot)$ with (A1)-(A3). Let us explain where (1.1)-(1.2) comes from. Consider the following reaction-diffusion equation:

$$
\begin{array}{r}
\frac{\tau}{\alpha} u_{t}=\Delta u+\frac{1}{\varepsilon^{2}}\left(u-u^{3}+\frac{\sqrt{2} \varepsilon}{3 \alpha} W(v)\right) \text { in } \mathbf{R}^{N}, t>0, \\
v_{t}=\Delta v+g(u, v), \\
u(x, 0)=u_{0}(x), \quad v(x, 0)=v_{0}(x) \text { in } \mathbf{R}^{N} .
\end{array}
$$

Here $\alpha>0$ is a given constant, while $\varepsilon>0$ is a small parameter. $W(v)$ is a monotone decreasing function with (A2). The term $\frac{\sqrt{2} \varepsilon}{3 \alpha} W(v)$ implies that the effect of an inhibitor is small enough so that it is balanced with the curvature effect of $\Gamma(t)$, where $\Gamma(t)=$ $\left\{x \in \mathbf{R}^{N} \mid u(x, t)=0\right\}$. If $[-1,1] \times[\underline{v}, \bar{v}]$ is included in some positively invariant region of (1.6), the solution of (1.6) stays in this region for all $t>0$, and then $W(v)$ remains bounded uniformly in $t>0$ whenever $\left(u_{0}, v_{0}\right)$ takes values in this region. 
By a similar argument as in [5], we can formally have

$$
\frac{\tau}{\alpha} V_{\Gamma(t)}=\frac{\sqrt{2}}{3 \alpha}(\psi(\infty)-\psi(-\infty))\left\|\psi^{\prime}\right\|_{L^{2}(\mathbf{R})}^{-2} W(v)-(N-1) H,
$$

which is equivalent to (1.1). Here $\psi(\gamma)$ is a solution of

$$
\begin{aligned}
\psi^{\prime \prime}(\gamma)+\psi(\gamma)-\psi(\gamma)^{3}=0, & -\infty<\gamma<\infty, \\
\psi(-\infty)=-1, & \psi(\infty)=1,
\end{aligned}
$$

and thus satisfies $\left\|\psi^{\prime}\right\|_{L^{2}(\mathbf{R})}^{2}=2 \sqrt{2} / 3$. Though this derivation is a formal one, X. Chen [2] showed that the interface $\Gamma(t)$ of $(1.6)$ is well-approximated by the solution of (1.1)-(1.2) by using a kind of super-subsolutions. Here $G^{ \pm}(v)=g( \pm 1, v)$. One can refer to [2, Remark 2.2].

By [3] and [2], it is proved that there exists a unique solution to (1.1)-(1.2) locally in time if $\Gamma_{0}$ is a compact surface of class $C^{2+\theta}$ with $0<\theta<1$ and $v_{0}(x)$ is of class $C^{1+\theta}$ with $\left\|v_{0}\right\|_{C^{1+\theta}\left(\mathbf{R}^{N}\right)}<\infty$.

Considering (1.1) $-(1.2)$ for every $\alpha>0$, we obtain the entire branching diagram of radially symmetric equilibrium solutions, which we call equilibrium balls.

For a first step to study the nonlinear stability of the equilibrium balls stated above, we study the linear stability of them. The nonlinear stability of the equilibrium solutions in (1.1)-(1.2) is yet to be proved. By studying the linearized eigenvalue problem, we show that the eigenvalues with nonnegative real parts are all real, and that they are characterized as values of a strictly convex function for specific discrete values of its argument. The stability is determined by the location of the zero points of this convex function. Using this fact, we present a criterion of stability in a useful form in Sec. 5 . We will show that stable equilibrium balls and unstable ones coexist near the saddle-node bifurcation points in the bifurcation diagram. If a given equilibrium ball is unstable and is located close to a saddle-node bifurcation point, instability occurs with spherically harmonic functions of low degrees. If a given equilibrium ball is located far from bifurcation points, it is unstable and the eigenfunction associated with the largest eigenvalue consists of spherically harmonic functions of high degrees. We will illustrate the situation described above in Sec. 6.

2. Radially symmetric equilibrium solutions and the linearized eigenvalue problem. Let $R>0$ be any positive number. We put $B(R)=\left\{x \in \mathbf{R}^{N}|| x \mid<R\right\}$ and $\Gamma(R)=\left\{x \in \mathbf{R}^{N}|| x \mid=R\right\}$. We seek a radially symmetric equilibrium solution $(\Gamma(R), v(r))$, where $r=|x|$. We have

$$
\begin{aligned}
\left.W(v(r))\right|_{r=R} & =(N-1) \alpha R^{-1} \\
\left(-\frac{d^{2}}{d r^{2}}-\frac{N-1}{r} \frac{d}{d r}\right) v & =G^{+}(v(r)) \chi_{\{r<R\}}+G^{-}(v(r)) \chi_{\{r>R\}} .
\end{aligned}
$$

We seek $v(|x|)$ so that $v(|\cdot|)$ belongs to $H^{1}\left(\mathbf{R}^{N}\right)$. From (A2), we can solve (2.1) as

$$
v(R)=U(R) \stackrel{\text { def }}{=} W^{-1}\left((N-1) \alpha R^{-1}\right) .
$$




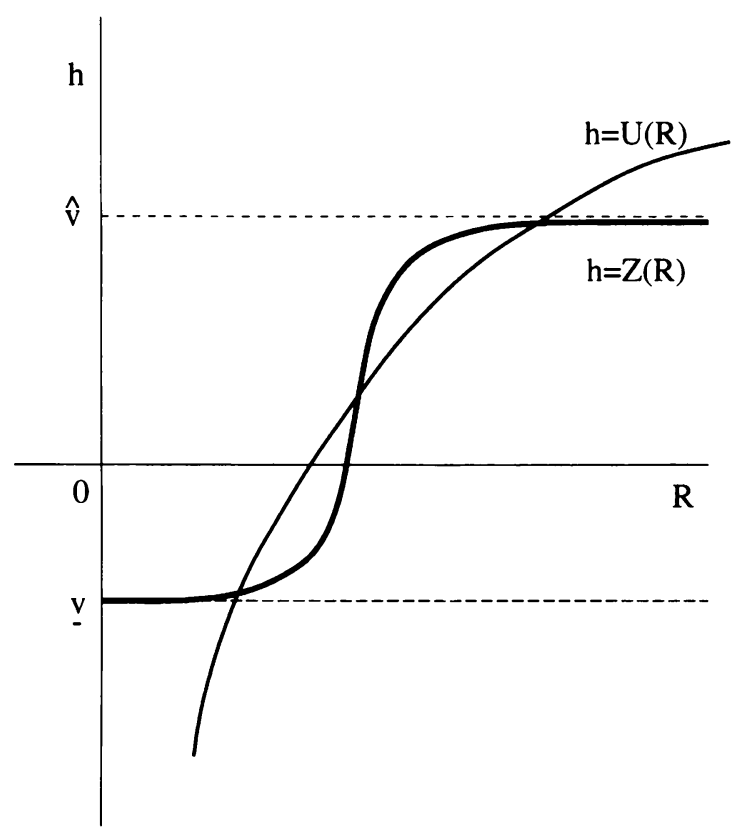

Fig. 1. The graphs of $Z(R)$ and $U(R)$

The solution for (2.2) is uniquely determined. We denote it by $V(r, R)$. It is given as a unique minimizer of a functional

$$
E[v]=\int_{0}^{\infty}\left[\frac{1}{2} r^{N-1}\left(v_{r}\right)^{2}+r^{N} G^{\mathrm{R}}(v(r), r)\right] d r
$$

for radially symmetric functions $v$ in $H^{1}\left(\mathbf{R}^{N}\right)$, where

$$
G^{\mathrm{R}}(v, r)=\int_{v}^{1} G^{+}(s) d s \chi_{\{r<R\}}-\int_{0}^{v} G^{-}(s) d s \chi_{\{r>R\}} .
$$

Lemma 1. ([4]) There exists a unique solution $v=V(r, R)$ to $(2.2)$. The solution $V(r, R)$ satisfies $V_{r}(r, R)<0$ with $V(+\infty, R)=0$. Define $Z(R) \stackrel{\text { def }}{=} V(R, R)$. Then $Z(R)$ satisfies

(1) $\frac{d Z}{d R}(R)>0$ for every $R \in(0, \infty)$;

(2) $\lim _{R \rightarrow+0} Z(R)=\underline{v}$ and $\lim _{R \rightarrow \infty} Z(R)=\widehat{v}$ hold true, where $\widehat{v} \in(\underline{v}, \bar{v})$ is a unique solution to

$$
\int_{\underline{v}}^{\widehat{v}} G^{-}(s) d s+\int_{\widehat{v}}^{\bar{v}} G^{+}(s) d s=0 .
$$

If $R \in(0, \infty)$ satisfies $Z(R)=U(R)$, then $(\Gamma(R), V(r, R))$ becomes a solution to $(2.1)$ and (2.2). Conversely, every solution to $(2.1)$ and $(2.2)$ is given by $(\Gamma(R), V(r, R)$ ) for some $R>0$ with $Z(R)=U(R)$. Therefore, solving (2.1)-(2.2) is equivalent to solving $Z(R)=U(R)$ for $R \in(0,+\infty)$.

One can see that the graph of $y=Z(R)$ is independent of $\alpha$, while that of $y=U(R)$ depends on $\alpha>0$ as in (2.3). Figure 1 shows a typical graph of $y=Z(R)$ and $y=U(R)$ 


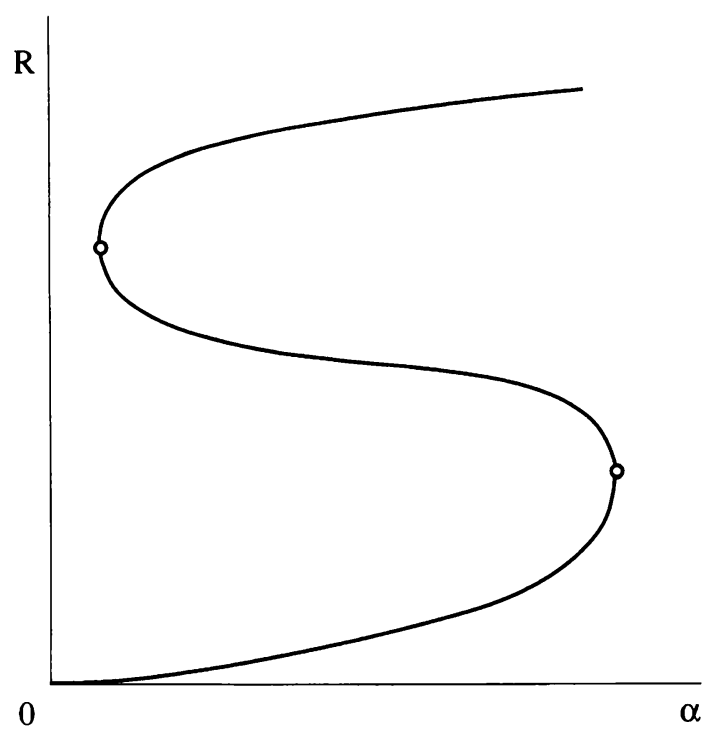

FIG. 2. The branching diagram of equilibrium balls

for some fixed $\alpha>0$. Example 1 in Sec. 6 is a concrete example of Fig. 1 . When $\alpha$ varies, the number of solutions of $Z(R)=U(R)$ changes. Figure 2 shows the bifurcation curve of equilibrium balls for $y=Z(R)$ and $y=U(R)$ given in Fig. 1. Our purpose in this paper is to clarify which part of the bifurcation curve corresponds to stable (or unstable) equilibrium balls, and to seek all of the eigenvalues for each equilibrium ball.

Consider an arbitrary intersection point of $y=Z(R)$ and $y=U(R)$, which we denote by $R_{0}$ hereafter. We have $Z\left(R_{0}\right)=U\left(R_{0}\right)$. We study the stability of an equilibrium ball $\left(\Gamma\left(R_{0}\right), V\left(r, R_{0}\right)\right)$ by the linearized eigenvalue problem.

We approximate solutions near $\left(\Gamma\left(R_{0}\right), V\left(r, R_{0}\right)\right)$ by putting

$$
\begin{aligned}
\Gamma(t) & =\left\{\left[R_{0}+\eta \rho(\xi) e^{\lambda t}\right] \xi \mid \xi \in S^{N-1}\right\} \\
v(x, t) & =V\left(|x|, R_{0}\right)+\eta w(x) e^{\lambda t}
\end{aligned}
$$

with infinitesimal $\eta$. Here $\lambda \in \mathbf{C}$, while $\rho(\xi)$ and $w(x)$ are functions on $S^{N-1}$ and $\mathbf{R}^{N}$, respectively. We substitute (2.4) into (1.1)-(1.2), and find $(\lambda, \rho, w)$ so that $\eta^{1}$-order terms balance.

Let us recall the spherically harmonic functions. Let $\Delta_{S^{N-1}}$ denote the LaplaceBeltrami operator on $S^{N-1}$. Consider an eigenvalue problem

$$
-\Delta_{S^{N-1}} \Phi=\kappa \Phi \quad \text { on } S^{N-1}
$$

for $\kappa \in \mathbf{R}$ and $\Phi(\xi) \in C\left(S^{N-1}\right)$. The set of eigenvalues is $\left\{\kappa_{n}=n(n+N-2) \mid n=\right.$ $0,1,2, \cdots\}$, and the eigenspace associated with $\kappa_{n}$ is the linear hull of all spherically harmonic functions of degree $n(n=0,1,2, \cdots)$. The set of the spherically harmonic functions is complete for the continuous functions on $S^{N-1}$.

We derive the linearized eigenvalue problem following [4]. Let $\Phi(\xi)$ be any fixed spherically harmonic function, and let $\kappa>0$ designate the associated eigenvalue of (2.5). 
We put $\rho(\xi)=\Phi(\xi)$. The $\eta^{1}$-order terms of (1.1) with (2.4) become

$$
\begin{gathered}
\tau \lambda e^{\lambda t} \Phi=e^{\lambda t} W^{\prime}\left(V\left(R_{0}, R_{0}\right)\right)\left(V_{r}\left(R_{0}, R_{0}\right) \Phi+\left.w(x)\right|_{|x|=R_{0}}\right) \\
+\alpha\left(R_{0}\right)^{-2}\left[\Delta_{S^{N-1}} \Phi+(N-1) \Phi\right] e^{\lambda t}
\end{gathered}
$$

This implies that $w(x)$ has the following form:

$$
w(x)=z(|x|) \Phi(x /|x|)
$$

with some $z(\cdot)$. The $\eta^{1}$-order terms of (1.2) with (2.4) and (2.7) become

$$
\begin{aligned}
z \Phi \lambda e^{\lambda t}- & e^{\lambda t} \Delta(z \Phi) \\
=G_{v}^{+} & \left(V\left(R_{0}, R_{0}\right)\right) z\left(R_{0}\right) e^{\lambda t} \Phi \chi_{\left\{r<R_{0}\right\}} \\
& +G_{v}^{-}\left(V\left(R_{0}, R_{0}\right)\right) z\left(R_{0}\right) e^{\lambda t} \Phi \chi_{\left\{r>R_{0}\right\}} \\
& +\Phi e^{\lambda t}\left[G^{+}\left(V\left(R_{0}, R_{0}\right)\right)-G^{-}\left(V\left(R_{0}, R_{0}\right)\right)\right] \delta_{R_{0}}(r) .
\end{aligned}
$$

Here $\delta_{R_{0}}(r)$ is the Dirac delta function concentrated at $r=R_{0}$. For any given $R>0$, we put

$$
\begin{array}{rcl}
p(R) & \stackrel{\text { def }}{=} & -W^{\prime}(V(R, R))>0, \\
Q(R) & \stackrel{\text { def }}{=} & G^{+}(V(R, R))-G^{-}(V(R, R))>0, \\
g(r, R) & \stackrel{\text { def }}{=} & -G_{v}^{+}(V(r, R)) \chi_{\{r<R\}}-G_{v}^{-}(V(r, R)) \chi_{\{r>R\}} .
\end{array}
$$

From (A1), we have $g(r, R)>0$ for all $r>0$ and $R>0$. Combining (2.6) and (2.8), we obtain the following problem: find $\lambda \in \mathbf{C}$ such that there exists $z(r) \not \equiv 0$ with

$$
\begin{array}{r}
\left(-\frac{d^{2}}{d r^{2}}-\frac{N-1}{r} \frac{d}{d r}+\frac{\kappa}{r^{2}}+g\left(r, R_{0}\right)+\lambda\right) z(r)=0, \quad r \in(0, \infty) \backslash\left\{R_{0}\right\} \\
-\left[z_{r}(r)\right]_{R_{0}-0}^{R_{0}+0}=Q\left(R_{0}\right) \\
-p\left(R_{0}\right) V_{r}\left(R_{0}, R_{0}\right)+\alpha(N-1-\kappa)\left(R_{0}\right)^{-2}-p\left(R_{0}\right) z\left(R_{0}\right)=\tau \lambda .
\end{array}
$$

Since the spherically harmonic functions are complete for $C\left(S^{N-1}\right)$, we can consider (2.9) for every $\kappa=n(n+N-2)(n=0,1,2, \cdots)$ to study the linearized problem for every $(\rho(\xi), w(x))$. If $(2.9)$ has a nontrivial solution $z(r)$ with some $\kappa=n(n+N-2), \lambda$ is an eigenvalue and $(\rho(\xi), w(x))=(\Phi(\xi), z(|x|) \Phi(x /|x|))$ is the associated eigenfunction. An equilibrium $\left(\Gamma\left(R_{0}\right), V\left(r, R_{0}\right)\right)$ is said to be stable in the linearized sense if there exist no eigenvalues in $\{\lambda \in \mathbf{C} \mid \operatorname{Re} \lambda \geq 0\}$, and to be unstable if there exists at least one eigenvalue in $\{\lambda \in \mathbf{C} \mid \operatorname{Re} \lambda>0\}$.

Hereafter we find $\lambda$ that belongs to $\{\lambda \in \mathbf{C} \mid \operatorname{Re} \lambda \geq 0\}$. We eliminate $z$ in (2.9) and find a relation free of $z$. Define

$$
\begin{aligned}
Y & =\left\{w(r) \in L^{2}(0, \infty) \mid\|w\|_{Y}^{2}=\int_{0}^{\infty} w(r)^{2} r^{N-1} d r<\infty\right\} \\
X_{\kappa} & =\left\{w(r) \in L^{2}(0, \infty) \mid\|w\|_{X_{\kappa}}^{2}=\int_{0}^{\infty}\left(w_{r}^{2}+w^{2}+\frac{\kappa}{r^{2}} w^{2}\right) r^{N-1} d r<\infty\right\} .
\end{aligned}
$$


Note that $X_{\kappa}$ and $X_{1}$ are equivalent spaces if $\kappa>0$. If no confusion occurs, we denote $X_{1}$ by $X$ for simplicity. For $w^{1}, w^{2} \in X_{\kappa}(\kappa \geq 0)$, we define a sesquilinear form

$$
B\left(w^{1}, w^{2}\right)=\int_{0}^{\infty}\left[w_{r}^{1} \overline{w_{r}^{2}}+\left(\frac{\kappa}{r^{2}}+g\left(r, R_{0}\right)+\lambda\right) w^{1} \overline{w^{2}}\right] r^{N-1} d r .
$$

Since $B(\cdot, \cdot)$ is bounded and coercive in $X_{\kappa}$, we apply the Lax-Milgram theorem to obtain a bounded linear operator

$$
L\left(\kappa, \lambda, R_{0}\right)^{-1}=\left(-\frac{d^{2}}{d r^{2}}-\frac{N-1}{r} \frac{d}{d r}+\frac{\kappa}{r^{2}}+g\left(r, R_{0}\right)+\lambda\right)^{-1}
$$

from $\left(X_{\kappa}\right)^{\prime}$ to $X_{\kappa}$. Here $\left(X_{\kappa}\right)^{\prime}$ is the anti-dual space of $X_{\kappa}$. For given $h \in\left(X_{\kappa}\right)^{\prime}$, we write $w=L\left(\kappa, \lambda, R_{0}\right)^{-1} h$ if and only if $B(w, u)=\langle h, u\rangle$ holds true for all $u \in X_{\kappa}$. Here $\langle h, u\rangle$ denotes the value of a functional $h$ acting on $u$. We have

$$
\langle h, u\rangle=\int_{0}^{\infty} h(r) \overline{u(r)} r^{N-1} d r
$$

if $h \in Y$. From (2.10), $L\left(\kappa, \lambda, R_{0}\right)^{-1}$ is bounded in $\mathcal{L}\left(\left(X_{\kappa}\right)^{\prime}, X_{\kappa}\right)$ uniformly in $\kappa \geq 0$, $\lambda \in\{\mu \in \mathbf{C} \mid \operatorname{Re} \mu \geq 0\}$ and $R_{0}>0$. Let $w(r, \kappa, \lambda)$ be defined as a unique solution of

$$
\begin{aligned}
-w_{r r}-\frac{N-1}{r} w_{r}+\left(\frac{\kappa}{r^{2}}+g\left(r, R_{0}\right)+\lambda\right) w & =0, \quad r \in(0, \infty) \backslash\left\{R_{0}\right\}, \\
-\left[w_{r}(r)\right]_{R_{0}-0}^{R_{0}+0} & =1 .
\end{aligned}
$$

We have $w(r, \kappa, \lambda)=L\left(\kappa, \lambda, R_{0}\right)^{-1} \delta_{R_{0}}$. We define

$$
\begin{array}{cl}
F(\alpha, \kappa, \lambda) \stackrel{\text { def }}{=} & -p\left(R_{0}\right) V_{r}\left(R_{0}, R_{0}\right)+(N-1-\kappa) \alpha\left(R_{0}\right)^{-2}-\tau \lambda \\
& -p\left(R_{0}\right) Q\left(R_{0}\right) w\left(R_{0}, \kappa, \lambda\right) .
\end{array}
$$

We have the following fact.

Lemma 2. ([4]) A complex number $\lambda \in\{\mu \in \mathbf{C} \mid \operatorname{Re} \mu \geq 0\}$ is an eigenvalue if and only if $F(\alpha, \kappa, \lambda)=0$ holds true with some $\kappa \in\{n(n+N-2) \mid n=0,1,2, \cdots\}$. The associated eigenfunction is given by $(\Phi(\xi), w(r, \kappa, \lambda) \Phi(\xi))$, where $\Phi$ is any spherically harmonic function of degree $n$ with $n(n+N-2)=\kappa$.

3. Distribution of the eigenvalues. Let $R_{0}>0$ satisfy $Z\left(R_{0}\right)=U\left(R_{0}\right)$, and we consider the linearized eigenvalue problem around $\left(\Gamma\left(R_{0}\right), V\left(r, R_{0}\right)\right)$. We show that the eigenvalues in the right half-plane $\{\lambda \in \mathbf{C} \mid \operatorname{Re} \lambda \geq 0\}$ are all real, and are given by $\left.\left\{\Lambda\left(\kappa, R_{0}\right)\right) \mid \kappa=n(n+N-2), n=0,1, \cdots\right\}$. Here $\Lambda\left(\kappa, R_{0}\right)$ is a real-valued and strictly upward convex function in $\kappa \in[0, \infty)$.

Define

$$
T\left(R_{0}\right) \stackrel{\text { def }}{=} p\left(R_{0}\right) Q\left(R_{0}\right)\left(R_{0}\right)^{-(N-1)} \int_{0}^{\infty} r^{N-1} w(r, 0,0)^{2} d r
$$

We have

LEMma 3. ([4]) Assume $\tau>T\left(R_{0}\right)$. If $\lambda$ has a nonnegative real part, $\lambda$ is a real number. Furthermore, $F_{\lambda}(\kappa, \lambda)<0$ holds true for all $\kappa \geq 0$ and real $\lambda \geq 0$.

We will show 
Lemma 4. $T\left(R_{0}\right)$ is uniformly bounded in $R_{0} \in(0,+\infty)$.

The preceding two lemmas imply that, if

$$
\tau>\tau_{*} \stackrel{\text { def }}{=} \sup _{R_{0}>0} T\left(R_{0}\right)
$$

then every eigenvalue with a nonnegative real part must be a real number.

Proof of Lemma 4. We denote $R_{0}$ by $R$ for simplicity. We define a positive constant $m$ as

$$
m^{2}=\min \left\{\min _{v \in[\underline{v}, \bar{v}]}\left(-G_{v}^{+}(v)\right), \min _{v \in[\underline{v}, \bar{v}]}\left(-G_{v}^{-}(v)\right)\right\}>0 .
$$

We have $g(r, R)>m^{2}>0$ for all $r>0$ and all $R>0$. Define $\zeta(r)$ by

$$
\begin{aligned}
-\zeta_{r r}-\frac{N-1}{r} \zeta_{r}+m^{2} \zeta & =0, \quad r \in(0, \infty) \backslash\{R\}, \\
-\left[z_{r}(r)\right]_{R-0}^{R+0} & =1 .
\end{aligned}
$$

Then $\zeta(r)$ is explicitly given by

$$
\zeta(r)= \begin{cases}R e^{-m R}(m r)^{-1} \sinh (m r) & \text { if } 0<r<R \\ (m r)^{-1} e^{-m r} R \sinh (m R) & \text { if } r>R\end{cases}
$$

when $N=3$, and

$$
\zeta(r)= \begin{cases}R K_{0}(m R) I_{0}(m r) & \text { if } 0<r<R \\ R I_{0}(m R) K_{0}(m r) & \text { if } r>R\end{cases}
$$

when $N=2$, respectively. Here $I_{0}$ and $K_{0}$ are modified Bessel functions.

We consider $\zeta(r)-w(r, 0,0)$. Then it satisfies

$$
\left\{-\frac{d}{d r}\left(r^{N-1} \frac{d}{d r} \cdot\right)+m^{2}\right\}(\zeta(r)-w(r, 0,0))=r^{N-1}\left[g(r, R)-m^{2}\right] z(r)>0 .
$$

Since the Green function of $-\frac{d}{d r}\left(r^{N-1} \frac{d}{d r} \cdot\right)+m^{2}$ is positive-valued, we obtain $w(r, 0,0)<$ $\zeta(r)$ for all $r>0$.

We have

$$
R^{-(N-1)} \int_{0}^{\infty} r^{N-1} w(r, 0,0)^{2} d r<R^{-(N-1)} \int_{0}^{\infty} r^{N-1} \zeta(r)^{2} d r
$$

Direct verification shows that

$$
R^{-(N-1)} \int_{0}^{\infty} r^{N-1} \zeta(r)^{2} d r=\frac{1}{4} m^{-3}\left(1-m R e^{-2 m R}-e^{-2 m R}\right)
$$

when $N=3$, and

$$
\begin{aligned}
& R^{-(N-1)} \int_{0}^{\infty} r^{N-1} \zeta(r)^{2} d r \\
& \quad=R K_{0}(m R)^{2} \int_{0}^{R} r I_{0}(m r)^{2} d r+R I_{0}(m R)^{2} \int_{R}^{\infty} r K_{0}(m r)^{2} d r
\end{aligned}
$$


when $N=2$. If $N=3$, the right-hand side remains bounded in $R \in(0, \infty)$. If $N=2$, using the properties of $I_{0}$ and $K_{0}$ (see [1]), we obtain

$$
\begin{aligned}
\lim _{R \rightarrow+0} R^{-1} \int_{0}^{\infty} r \zeta(r)^{2} d r & =0 \\
\lim _{R \rightarrow+\infty} R^{-1} \int_{0}^{\infty} r \zeta(r)^{2} d r & =\frac{1}{4} m^{-3}
\end{aligned}
$$

Hence in both cases, $R^{-(N-1)} \int_{0}^{\infty} r^{N-1} \zeta(r)^{2} d r$ remains bounded uniformly in $R \in(0, \infty)$. This completes the proof of Lemma 4 .

Combining Lemmas 3 and 4 , we can solve $F(\alpha, \kappa, \lambda)=0$ since $\lambda=\Lambda\left(\kappa, R_{0}\right)$ by the implicit function theorem. A function $\Lambda\left(\kappa, R_{0}\right)$ is a real-valued function of $\kappa$ in some interval of definition. By a similar argument as in [4], we find that $\Lambda\left(\kappa, R_{0}\right)$ is a strictly upward convex function with $\Lambda\left(N-1, R_{0}\right)=0$. Indeed, we have

$$
\left(-\frac{d^{2}}{d r^{2}}-\frac{N-1}{r} \frac{d}{d r}+\frac{N-1}{r^{2}}+g\left(r, R_{0}\right)\right)\left(-V_{r}\left(r, R_{0}\right)\right)=Q\left(R_{0}\right) \delta_{R_{0}}(r),
$$

which implies that $F(\alpha, N-1,0)=0$ and thus $\Lambda\left(N-1, R_{0}\right)=0$. This fact is clear because a translation of $\left(\Gamma\left(R_{0}\right), V\left(r, R_{0}\right)\right)$ gives another solution of (2.1) and (2.2). By direct calculation, one can show that $L\left(\kappa, \lambda, R_{0}\right)^{-1}$ is selfadjoint and is infinitely many times continuously differentiable by $\kappa>0$ and $\lambda \in[0,+\infty)$ in $\mathcal{L}\left(X^{\prime}, X\right)$. Here we write $X$ instead of $X_{1}$ for simplicity. We have

$$
\begin{aligned}
& \frac{\partial}{\partial \lambda}\left(L\left(\kappa, \lambda, R_{0}\right)^{-1}\right)=-L\left(\kappa, \lambda, R_{0}\right)^{-2}, \\
& \frac{\partial}{\partial \kappa}\left(L\left(\kappa, \lambda, R_{0}\right)^{-1}\right)=-L\left(\kappa, \lambda, R_{0}\right)^{-1}\left(r^{-2} L\left(\kappa, \lambda, R_{0}\right)^{-1}\right)
\end{aligned}
$$

in $\mathcal{L}\left(X^{\prime}, X\right)$ for every $\kappa>0$ and $\lambda \in[0,+\infty)$. Direct calculation yields

$$
\Lambda_{\kappa \kappa}=\left(F_{\lambda \lambda}(\kappa, \Lambda)\left(\Lambda_{\kappa}\right)^{2}+2 F_{\kappa \lambda}(\kappa, \Lambda) \Lambda_{\kappa}+F_{\kappa \kappa}(\kappa, \Lambda)\right) /\left(-F_{\lambda}(\kappa, \Lambda)\right) .
$$

Using (3.2) and $w(r, \kappa, \lambda)=L\left(\kappa, \lambda, R_{0}\right)^{-1} \delta_{R_{0}}$, we have

$$
\begin{aligned}
& w_{\lambda}(r, \kappa, \lambda)=-L\left(\kappa, \lambda, R_{0}\right)^{-2} \delta_{R_{0}} \\
& w_{\kappa}(r, \kappa, \lambda)=-L\left(\kappa, \lambda, R_{0}\right)^{-1}\left(r^{-2} L\left(\kappa, \lambda, R_{0}\right)^{-1} \delta_{R_{0}}\right)
\end{aligned}
$$

in $X$. From $w\left(R_{0}, \kappa, \lambda\right)={ }_{X}\left\langle w(r, \kappa, \lambda), \delta_{R_{0}}\right\rangle_{X^{\prime}}$, we obtain

$$
\begin{aligned}
w_{\lambda}\left(R_{0}, \kappa, \lambda\right) & =\left\langle-L^{-2} \delta_{R_{0}}, \delta_{R_{0}}\right\rangle=-\left(L^{-1} \delta_{R_{0}}, L^{-1} \delta_{R_{0}}\right)_{Y} \\
w_{\kappa}\left(R_{0}, \kappa, \lambda\right) & =\left\langle-L^{-1}\left(r^{-2} L^{-1} \delta_{R_{0}}\right), \delta_{R_{0}}\right\rangle \\
& =-\left(r^{-1} L^{-1} \delta_{R_{0}}, r^{-1} L^{-1} \delta_{R_{0}}\right)_{Y}
\end{aligned}
$$


with $L^{-1}=L\left(\kappa, \lambda, R_{0}\right)^{-1} \in \mathcal{L}\left(X^{\prime}, X\right)$. Consequently we obtain

$$
\begin{aligned}
w_{\lambda \lambda}\left(R_{0}, \kappa, \lambda\right) & =2\left(L^{-2} \delta_{R_{0}}, L^{-1} \delta_{R_{0}}\right)_{Y} \\
& =2\left(w_{\lambda}(r, \kappa, \lambda), L\left(\kappa, \lambda, R_{0}\right) w_{\lambda}(r, \kappa, \lambda)\right)_{Y} \\
& =2 B\left(w_{\lambda}(r, \kappa, \lambda), w_{\lambda}(r, \kappa, \lambda)\right), \\
w_{\kappa \lambda}\left(R_{0}, \kappa, \lambda\right) & =2\left(L^{-1}\left(r^{-2} L^{-1} \delta_{R_{0}}\right), L^{-1} \delta_{R_{0}}\right)_{Y} \\
& =2\left(w_{\kappa}, L w_{\lambda}\right)_{Y}=2 B\left(w_{\kappa}, w_{\lambda}\right), \\
w_{\kappa \kappa}\left(R_{0}, \kappa, \lambda\right) & =2\left(r^{-1} L^{-1}\left(r^{-2} L^{-1} \delta_{R_{0}}\right), r^{-1} L^{-1} \delta_{R_{0}}\right)_{Y} \\
& =2\left(w_{\kappa}, L w_{\kappa}\right)_{Y}=2 B\left(w_{\kappa}, w_{\kappa}\right) .
\end{aligned}
$$

Using the Schwarz inequality and the linear independence of $w_{\lambda}$ and $w_{\kappa}$, we obtain $\left|B\left(w_{\kappa}, w_{\lambda}\right)\right|<B\left(w_{\lambda}, w_{\lambda}\right) B\left(w_{\kappa}, w_{\kappa}\right)$. Using this fact and the definition of $F(\alpha, \kappa, \lambda)$, we get $F_{\lambda \lambda} F_{\kappa \kappa}-\left(F_{\lambda \kappa}\right)^{2}>0$. Combining this inequality and Lemma 3 , we obtain

$$
\Lambda_{\kappa \kappa}\left(\kappa, R_{0}\right)<0
$$

for every $\kappa$ in the interval of definition.

Since $w\left(R_{0}, \kappa, \lambda\right)>0$, we find that $\Lambda\left(\kappa, R_{0}\right)<0$ in $\kappa>N-1+\alpha^{-1}\left(R_{0}\right)^{2} p\left(R_{0}\right)\left(-V_{r}\left(R_{0}\right.\right.$, $\left.R_{0}\right)$ ). Combining this fact, $\Lambda\left(N-1, R_{0}\right)=0$ and $\Lambda_{\kappa \kappa}\left(\kappa, R_{0}\right)<0$, we have the following three cases corresponding to the sign of $\Lambda\left(0, R_{0}\right)$. See Fig. 3 .

(1) (Case 1) $\Lambda\left(\kappa, R_{0}\right)$ satisfies $\Lambda\left(0, R_{0}\right)>0$ and $\Lambda\left(N-1, R_{0}\right)=0$. It follows that

$$
\begin{aligned}
& \Lambda\left(\kappa, R_{0}\right)>0 \text { if } \kappa \in[0, N-1), \\
& \Lambda\left(\kappa, R_{0}\right)<0 \text { if } \kappa \in(N-1,+\infty) .
\end{aligned}
$$

(2) (Case 2) $\Lambda\left(\kappa, R_{0}\right)$ satisfies $\Lambda\left(0, R_{0}\right)<0$ and $\Lambda\left(N-1, R_{0}\right)=0$. There exists a unique $\kappa^{*} \in\left(N-1, N-1+\alpha^{-1}\left(R_{0}\right)^{2} p\left(R_{0}\right)\left(-V_{r}\left(R_{0}, R_{0}\right)\right)\right)$ that satisfies $\Lambda\left(\kappa^{*}, R_{0}\right)=0$. It follows that

$$
\begin{aligned}
& \Lambda\left(\kappa, R_{0}\right)<0 \text { if } \kappa \in[0, N-1), \\
& \Lambda\left(\kappa, R_{0}\right)>0 \text { if } \kappa \in\left(N-1, \kappa^{*}\right), \\
& \Lambda\left(\kappa, R_{0}\right)<0 \text { if } \kappa \in\left(\kappa^{*},+\infty\right) .
\end{aligned}
$$

(3) (Case 3) $\Lambda\left(\kappa, R_{0}\right)$ satisfies $\Lambda\left(0, R_{0}\right)=0$ and $\Lambda\left(N-1, R_{0}\right)=0$. It follows that

$$
\begin{aligned}
& \Lambda\left(\kappa, R_{0}\right)>0 \text { if } \kappa \in(0, N-1), \\
& \Lambda\left(\kappa, R_{0}\right)<0 \text { if } \kappa \in(N-1, \infty) .
\end{aligned}
$$

We have thus obtained the following assertion.

LEMmA 5. $\lambda=\Lambda\left(\kappa, R_{0}\right)$ is a strictly upward convex function and satisfies one of the three cases: (Case 1), (Case 2) and (Case 3) stated above.

Proof. Combining $\Lambda\left(N-1, R_{0}\right)=0$ and $\Lambda_{\kappa \kappa}\left(\kappa, R_{0}\right)<0$, we obtain the three cases following the sign of $\Lambda\left(0, R_{0}\right)$.

Note that Case 3 corresponds to a saddle-node bifurcation point. We can differentiate the bifurcation curve in Fig. 2 by $R$ at a turning point, and obtain an eigenfunction associated with zero eigenvalues in the linearized eigenvalue problem. For other points 


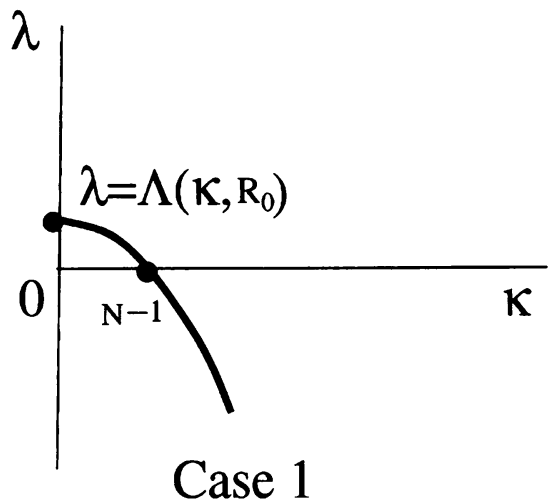

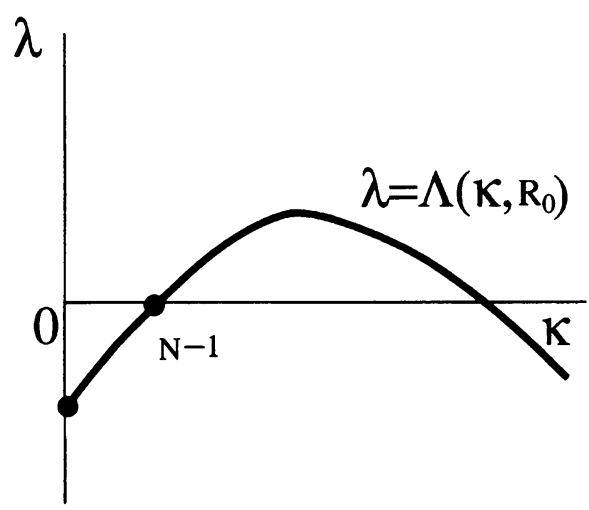

Case 2

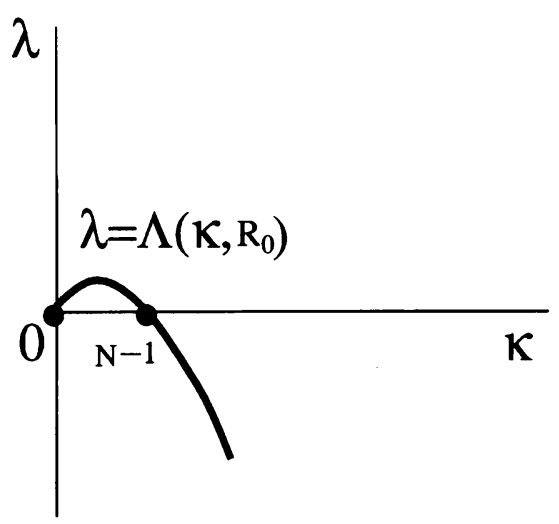

Case 3

FIG. 3. The graph of $\lambda=\Lambda\left(\kappa, R_{0}\right)$

except them, Case 1 or Case 2 appear following $\frac{d Z}{d R}\left(R_{0}\right)<\frac{d U}{d R}\left(R_{0}\right)$ and $\frac{d Z}{d R}\left(R_{0}\right)>\frac{d U}{d R}\left(R_{0}\right)$, respectively. We will discuss this situation in the next section.

4. Stability for radially symmetric perturbations. Let $R_{0}$ be an arbitrary number with $Z\left(R_{0}\right)=U\left(R_{0}\right)$, and let $\left(\Gamma\left(R_{0}\right), V\left(r, R_{0}\right)\right)$ be the associated equilibrium ball. We study the stability of $\left(\Gamma\left(R_{0}\right), V\left(r, R_{0}\right)\right)$ for radially symmetric small perturbations. For this purpose we study the sign of $\Lambda\left(0, R_{0}\right)$.

LEMma 6. The following relation

$$
\frac{d Z}{d R}\left(R_{0}\right)-\frac{d U}{d R}\left(R_{0}\right)=W^{\prime}\left(V\left(R_{0}, R_{0}\right)\right)^{-1} F(\alpha, 0,0)
$$

holds true.

Proof. For any $R>0, U(R)=W^{-1}((N-1) \alpha / R)$ is differentiable with

$$
\frac{d U}{d R}(R)=-W^{\prime}(U(R))^{-1} \frac{(N-1) \alpha}{R^{2}} .
$$


Since $V\left(R_{0}, R_{0}\right)=U\left(R_{0}\right)$, we have

$$
\frac{d U}{d R}\left(R_{0}\right)=-\frac{(N-1) \alpha}{W^{\prime}\left(V\left(R_{0}, R_{0}\right)\right) R_{0}^{2}}=\frac{(N-1) \alpha}{p\left(R_{0}\right) R_{0}^{2}} .
$$

For every $R>0, Z(R)$ is defined by $Z(R)=V(R, R)$, where $V(r, R)$ satisfies (2.2). Differentiating $(2.2)$ by $R$, we obtain

$$
\begin{aligned}
\left(-\frac{d^{2}}{d r^{2}}-\frac{N-1}{r} \frac{d}{d r}\right) V_{R}= & G^{+}(V(r, R)) V_{R} \chi_{\{r<R\}}+G^{-}(V(r, R)) V_{R} \chi_{\{r>R\}} \\
& +G^{+}(V(R, R)) \delta_{R}(r)-G^{-}(V(R, R)) \delta_{R}(r),
\end{aligned}
$$

and thus

$$
V_{R}(r, R)=Q(R)\left(-\frac{d^{2}}{d r^{2}}-\frac{N-1}{r} \frac{d}{d r}+g(r, R)\right)^{-1} \delta_{R}
$$

for every $R>0$. For all $R>0$, we have

$$
\frac{d Z}{d R}(R)=V_{r}(R, R)+V_{R}(R, R)
$$

Using $V_{R}\left(r, R_{0}\right)=Q\left(R_{0}\right) w(r, 0,0)$, we have

$$
\frac{d Z}{d R}\left(R_{0}\right)-\frac{d U}{d R}\left(R_{0}\right)=V_{r}\left(R_{0}, R_{0}\right)+Q\left(R_{0}\right) w\left(R_{0}, 0,0\right)-\frac{(N-1) \alpha}{p\left(R_{0}\right) R_{0}^{2}}
$$

The right-hand side equals $-p\left(R_{0}\right)^{-1} F(\alpha, 0,0)$. Using the definition of $p\left(R_{0}\right)$, we obtain the desired relation.

The stability for radially symmetric perturbations follows from Lemma 6 .

LEMmA 7. If $\frac{d Z}{d R}\left(R_{0}\right)-\frac{d U}{d R}\left(R_{0}\right)<0$, then $\Lambda\left(0, R_{0}\right)>0$ holds true and thus $\left(\Gamma\left(R_{0}\right)\right.$, $\left.V\left(r, R_{0}\right)\right)$ is unstable for radially symmetric perturbations. If $\frac{d Z}{d R}\left(R_{0}\right)-\frac{d U}{d R}\left(R_{0}\right)>0$, then $\Lambda\left(0, R_{0}\right)<0$ and thus $\left(\Gamma\left(R_{0}\right), V\left(r, R_{0}\right)\right)$ is stable for radially symmetric perturbations.

Proof. To study the stability for radially symmetric perturbations, we have to study $F(\alpha, \kappa, \lambda)=0$ when $\kappa=0$. Using $F\left(\alpha, 0, \Lambda\left(0, R_{0}\right)\right)=0$ and $F_{\lambda}(\alpha, 0, \lambda)<0$ for $\lambda \geq 0$, we find that $\Lambda\left(0, R_{0}\right)>0$ holds true if $F(\alpha, 0,0)>0$, and $\Lambda(\alpha, 0)<0$ if $F(\alpha, 0,0)<0$, respectively. From Lemma 6 and $W^{\prime}\left(V\left(R_{0}, R_{0}\right)\right)<0$, we find that $\Lambda\left(0, R_{0}\right)>0$ holds if $\frac{d Z}{d R}\left(R_{0}\right)-\frac{d U}{d R}\left(R_{0}\right)<0$, and $\Lambda\left(0, R_{0}\right)<0$ holds if $\frac{d Z}{d R}\left(R_{0}\right)-\frac{d U}{d R}\left(R_{0}\right)>0$. This completes the proof.

5. Stability for general perturbations. In this section, we study the stability of $\left(\Gamma\left(R_{0}\right), V\left(r, R_{0}\right)\right)$ for general perturbations including non-radial ones. We assume that $Z\left(R_{0}\right)=U\left(R_{0}\right)$ and $\frac{d Z}{d R}\left(R_{0}\right)>\frac{d U}{d R}\left(R_{0}\right)$. In this case, $\left(\Gamma\left(R_{0}\right), V\left(r, R_{0}\right)\right)$ is stable for radially symmetric perturbations, and Case 2 in Sec. 3 holds true.

First we study the sign of $\Lambda\left(2 N, R_{0}\right)$. We express $F(\alpha, 2 N, 0)$ in a more useful form. We will define a function of $R \in(0, \infty)$, say $f(R)$, and write $F(\alpha, 2 N, 0)$ as $f\left(R_{0}\right)$. We define

$$
\begin{aligned}
& w_{1}(r, R)=L(N-1,0, R)^{-1} \delta_{R} \\
& w_{2}(r, R)=L(2 N, 0, R)^{-1} \delta_{R}
\end{aligned}
$$


in $X_{1}$ for each $R>0$. Differentiating $(2.2)$ by $r$, we have

$$
-V_{r}(r, R)=Q(R) w_{1}(r, R) .
$$

From (2.1), we get $\alpha=(N-1)^{-1} R W(V(R, R))$. Substituting these into the definition of $F(\alpha, 2 N, 0)$, we obtain

$$
F(\alpha, 2 N, 0)=f\left(R_{0}\right)
$$

where $f(R)$ is defined by

$$
f(R) \stackrel{\text { def }}{=}-\frac{N+1}{N-1} \frac{W(V(R, R))}{R}+p(R) Q(R)\left(w_{1}(R, R)-w_{2}(R, R)\right)
$$

for all $R>0$. We have

$$
w_{1}(R, R)-w_{2}(R, R)=R^{-(N-1)}\left\langle\delta_{R}, w_{1}(r, R)-w_{2}(r, R)\right\rangle .
$$

Since

$$
w_{1}(r, R)-w_{2}(r, R)=(N+1) L(N-1,0, R)^{-1}\left(r^{-2} L(2 N, 0, R)^{-1} \delta_{R}\right),
$$

and $L(\kappa, 0, R)^{-1}$ is selfadjoint, we get

$$
w_{1}(R, R)-w_{2}(R, R)=(N+1) \int_{0}^{\infty} r^{N-3} w_{1}(r, R) w_{2}(r, R) d r
$$

Substituting this relation into (5.3), we obtain

$$
\frac{1}{N+1} f(R)=-\frac{W(V(R, R))}{(N-1) R}+\frac{p(R) Q(R)}{R^{N-1}} \int_{0}^{\infty} r^{N-3} w_{1}(r, R) w_{2}(r, R) d r
$$

for all $R>0$. We have the following assertion.

Theorem 1. Fix $R_{0}>0$ so that $Z\left(R_{0}\right)=U\left(R_{0}\right)$ and $\frac{d Z}{d R}\left(R_{0}\right)>\frac{d U}{d R}\left(R_{0}\right)$ hold true. Then an equilibrium solution $\left(\Gamma\left(R_{0}\right), V\left(r, R_{0}\right)\right)$ of $(1.1)-(1.2)$ is stable if $f\left(R_{0}\right)<0$, and is unstable if $f\left(R_{0}\right)>0$. Here $f$ is a real-valued function given by (5.3).

Proof. We remark that $\kappa$ takes only discrete values $\left\{\kappa_{n}=n(n+N-2) \mid n=0,1, \cdots\right\}$. If $f\left(R_{0}\right)>0$, then $F(\alpha, 2 N, 0)>0$ holds true and hence $\Lambda\left(2 N, R_{0}\right)>0$ holds true. Since the linearized eigenvalue problem at $\left(\Gamma\left(R_{0}\right), V\left(r, R_{0}\right)\right)$ has at least one positive eigenvalue, $\left(\Gamma\left(R_{0}\right), V\left(r, R_{0}\right)\right)$ is unstable. On the other hand, assume $f\left(R_{0}\right)<0$. Then $\Lambda\left(\kappa_{n}, R_{0}\right)<0$ for $n \neq 1$, because $\Lambda\left(\kappa, R_{0}\right)$ is a strictly upward convex function with Case 2 in Lemma 5. The zero eigenvalue $0=\Lambda\left(N-1, R_{0}\right)$ is always associated with the transition in space, and all other eigenvalues lie in $\{\lambda \in \mathbf{C} \mid \operatorname{Re} \lambda<0\}$. Therefore $\left(\Gamma\left(R_{0}\right), V\left(r, R_{0}\right)\right)$ is stable.

One will notice that (5.3) is a function that depends only on $R$ and is independent of $\alpha$.

Remark 1. We consider Case 2 in Sec. 3 when $f\left(R_{0}\right)>0 . \quad \lambda=\Lambda\left(\kappa, R_{0}\right)$ has a unique maximizer, say, $\widehat{\kappa}$. This $\widehat{\kappa}$ satisfies $\Lambda_{\kappa}\left(\widehat{\kappa}, \Lambda\left(\widehat{\kappa}, R_{0}\right)\right)=0$, and hence it satisfies $F_{\kappa}\left(\widehat{\kappa}, \Lambda\left(\widehat{\kappa}, R_{0}\right)\right)=0$. In other words, $\widehat{\kappa}>0$ is uniquely determined by

$$
-\frac{\alpha}{R_{0}^{2}}+p\left(R_{0}\right) Q\left(R_{0}\right) \int_{0}^{\infty} r^{N-3} w\left(r, \widehat{\kappa}, \Lambda\left(\widehat{\kappa}, R_{0}\right)\right)^{2} d r=0 .
$$

This relation characterizes the $\widehat{\kappa}$ that gives the largest eigenvalue. 
Let $\left(R_{*}, \alpha_{*}\right)$ be an arbitrary saddle-node bifurcation point, that is,

$$
Z\left(R_{*}\right)=U\left(R_{*}\right), \quad \frac{d Z}{d R}\left(R_{*}\right)=\frac{d U}{d R}\left(R_{*}\right) .
$$

Then Case 3 is valid for $\left(\Gamma\left(R_{*}\right), V\left(r, R_{*}\right)\right)$ when $\alpha=\alpha_{*}$. We have $f\left(R_{*}\right)<0$ from $\Lambda\left(2 N, R_{*}\right)<0$ and the proof of Theorem 1 . We have the following assertion, for which we give the proof later in this section.

LEMMA 8. $f(R)$ is continuous in $R>0$.

Using this fact and $f\left(R_{*}\right)<0$, we obtain the following assertion.

Theorem 2. Fix any $R_{*}>0$ with $Z\left(R_{*}\right)=U\left(R_{*}\right)$ and $\frac{d Z}{d R}\left(R_{*}\right)=\frac{d U}{d R}\left(R_{*}\right)$. If $\left|R_{0}-R_{*}\right|$ is small enough, then $f\left(R_{0}\right)<0$ holds true. Assume $\frac{d Z}{d R}\left(R_{0}\right)>\frac{d U}{d R}\left(R_{0}\right)$ in addition. Then $\left(\Gamma\left(R_{0}\right), V\left(r, R_{0}\right)\right)$ is a stable equilibrium solution of (1.1) and (1.2).

Proof. The assertion follows directly from Theorem 1 and Lemma 8.

Theorem 2 implies that there exists a branch associated with stable equilibrium balls near a saddle-node bifurcation point.

Proof of Lemma 8. Putting $r=R s$ and $u(s, R)=V(R s, R)$, we obtain

$$
\left(-\frac{d^{2}}{d s^{2}}-\frac{N-1}{s} \frac{d}{d s}\right) u=R^{2} G(u, s) \stackrel{\text { def }}{=} R^{2}\left[G^{+}(u) \chi_{\{(0,1)\}}+G^{-}(u) \chi_{\{(1, \infty)\}}\right] .
$$

Differentiating by $R$, we have

$$
\left(-\frac{d^{2}}{d s^{2}}-\frac{N-1}{s} \frac{d}{d s}-R^{2} G_{v}(u, s)\right) u_{R}=2 R G(u, s) \text { in } \mathbf{R}^{N} .
$$

We see that $u_{R}(s, R)$ belongs to $X_{1}$. Indeed, $G(u(s, R), s)$ belongs to $X_{1}$, and $-R^{2} G_{v}(u(s, R), s)$ is positive and away from 0 uniformly in $s>0$. Hence (5.7) implies $u_{R}(s, R)$ belongs to $X_{1}$. We define $z(s, R, \kappa)=w(r, R, \kappa)$ for all $R>0, \kappa \geq 0$. Then $z(s, R, \kappa)$ satisfies

$$
\begin{aligned}
\left(-\frac{d^{2}}{d s^{2}}-\frac{N-1}{s} \frac{d}{d s}+\frac{\kappa}{s^{2}}+h(s, R)\right) z(s, R, \kappa) & =0, \quad s \in(0, \infty) \backslash\{1\}, \\
-\left[z_{s}(s, R, \kappa)\right]_{1-0}^{1+0} & =R .
\end{aligned}
$$

Here we put $h(s, R)=R^{2} g(R s, R)$. We define a bounded linear operator

$$
L_{s}(\kappa, R)^{-1} \stackrel{\text { def }}{=}\left(-\frac{d^{2}}{d s^{2}}-\frac{N-1}{s} \frac{d}{d s}+\frac{\kappa}{s^{2}}+h(s, R)\right)^{-1}
$$

from $\left(X_{1}\right)^{\prime}$ to $X_{1}$ in a similar way as for $L(\kappa, \lambda, R)^{-1}$ in Sec. 2. For any fixed $\kappa>$ $0, L_{s}(\kappa, R)^{-1}$ is bounded in $\mathcal{L}\left(\left(X_{1}\right)^{\prime}, X_{1}\right)$ uniformly in $R>0$. We have $z(s, R, \kappa)=$ $R L_{s}(\kappa, R)^{-1} \delta_{1}$. Using $z(s, R, \kappa)$, we express $f(R)$ as

$$
f(R)=-\frac{(N+1) W(V(R, R))}{(N-1) R}+p(R) Q(R)[z(1, R, N-1)-z(1, R, 2 N)] .
$$

Since $V(R, R), p(R)$, and $Q(R)$ are continuous in $R>0$ by virtue of (A1), (A2), and Lemma 1 , it suffices to show that $z(1, R, \kappa)$ is continuous in $R>0$ for fixed $\kappa>0$. It 
suffices to prove that $\left\langle L_{s}(\kappa, R)^{-1} \delta_{1}, \delta_{1}\right\rangle$ is continuous in $R>0$. For $R_{1}, R_{2}>0$, we have

$$
\begin{aligned}
\left\langle L_{s}\left(\kappa, R_{2}\right)^{-1} \delta_{1}, \delta_{1}\right\rangle-\left\langle L_{s}\left(\kappa, R_{1}\right)^{-1} \delta_{1}, \delta_{1}\right\rangle \\
\quad=\left\langle L_{s}\left(\kappa, R_{2}\right)^{-1}\left(h\left(s, R_{1}\right)-h\left(s, R_{2}\right)\right) L_{s}\left(\kappa, R_{1}\right)^{-1} \delta_{1}, \delta_{1}\right\rangle \\
\quad=\int_{0}^{\infty}\left(h\left(s, R_{1}\right)-h\left(s, R_{2}\right)\right)\left(L_{s}\left(\kappa, R_{2}\right)^{-1} \delta_{1}\right)\left(L_{s}\left(\kappa, R_{1}\right)^{-1} \delta_{1}\right) s^{N-1} d s
\end{aligned}
$$

For any fixed $\kappa>0, L_{s}(\kappa, R)^{-1} \delta_{1}$ remains bounded in $X_{1}$ uniformly in $R>0$, and then Lebesgue's dominated convergence theorem implies that the right-hand side goes to 0 as $R_{2} \rightarrow R_{1}$. This completes the proof.

REMARK 2. If $G^{ \pm}(v)$ and $W(v)$ belong to $C^{2}[\underline{v}, \bar{v}]$, then $f(R)$ belongs to $C^{1}(0, \infty)$. Indeed, $V(R, R)=u(1, R)$ is continuously differentiable in $R>0$ from (5.7) and (5.6). It suffices to prove that $z(1, R, \kappa)$ is continuously differentiable in $R>0$. We have

$$
\frac{\partial}{\partial R} L_{s}(R, \kappa)^{-1}=-L_{s}(\kappa, R)\left[h_{R}(s, R) L_{s}(\kappa, R)^{-1}\right]
$$

in $\mathcal{L}\left(\left(X_{1}\right)^{\prime}, X_{1}\right)$. Write

$$
z(1, R, \kappa)=\left\langle z(s, R, \kappa), \delta_{1}\right\rangle=\left\langle R L_{s}(\kappa, R)^{-1} \delta_{1}, \delta_{1}\right\rangle
$$

Since $L_{s}(\kappa, R)^{-1}$ is selfadjoint, we have

$$
\begin{aligned}
& \left(\left\langle R L_{s}(\kappa, R)^{-1} \delta_{1}, \delta_{1}\right\rangle\right)_{R} \\
& \quad=\left\langle L_{s}(\kappa, R)^{-1} \delta_{1}, \delta_{1}\right\rangle-R \int_{0}^{\infty} h_{R}(s, R)\left(L_{s}(\kappa, R)^{-1} \delta_{1}\right)^{2} s^{N-1} d s \\
& \quad=R^{-1} z(1, R, \kappa)-R^{-1} \int_{0}^{\infty} h_{R}(s, R) z(s, R, \kappa)^{2} s^{N-1} d s .
\end{aligned}
$$

After simple calculation, we have

$$
\begin{aligned}
h_{R}(s, R)= & 2 R G(u(s, R), s) \\
& -R^{2}\left[G_{v v}^{+}(u(s, R)) \chi_{\{s<1\}}+G_{v v}^{-}(u(s, R)) \chi_{\{s>1\}}\right] u_{R}(s, R) .
\end{aligned}
$$

Because $u(s, R)-\underline{v}$ and $u_{R}(s, R)$ belong to $X_{1}, h_{R}(s, R)$ belongs to $X_{1}$. The right-hand side of (5.8) exists and depends continuously on $R>0$ since $G^{ \pm}(\cdot)$ belong to $C^{2}[\underline{v}, \bar{v}]$. Thus $z(1, R, \kappa)$ is continuously differentiable in $R$, and hence $f(R)$ is of class $C^{1}(0, \infty)$.

6. Examples. In this section, we present two examples to illustrate the multiple existence and stability of equilibrium balls. If an equilibrium ball is sufficiently close to a bifurcation point, the stability is determined by eigenvalues associated with $\kappa=$ $0,2 N$. When it is unstable, the instability occurs with low modes, more precisely, with $\kappa=0,2 N$. On the contrary, if an equilibrium ball is far from the bifurcation points, it is unstable with high modes, more precisely, with large $\kappa>0$. We illustrate these situations by typical examples. 
6.1. Example 1. Consider, for $N=3$, the following problem:

$$
\begin{aligned}
\tau V_{\Gamma(t)}=\frac{a_{2}}{v+a_{1}}-2 \alpha H & \text { on } \Gamma(t), t>0, \\
v_{t}-\Delta v=\left(1-b^{2} v+c\right) \chi_{\Omega^{+}(t)}+\left(-1-b^{2} v+c\right) \chi_{\Omega^{-}(t)} & \text { in } \mathbf{R}^{3}, t>0 .
\end{aligned}
$$

Here $a_{1}, a_{2}, b$ and $c$ are positive constants with $b^{2} a_{1}+c>1$. The associated reactiondiffusion equation is (1.6) with $W(v)=a_{1} /\left(v+a_{2}\right)$. Equation (6.1)-(6.2) satisfies all assumptions (A1), (A2), and (A3) in Sec. 1 with

$$
\underline{v}=-b^{-2}(1-c), \quad \widehat{v}=b^{-2} c, \quad \bar{v}=b^{-2}(1+c) .
$$

We have

$$
U(R)=\frac{a_{2} R}{2 \alpha}-a_{1}
$$

We can explicitly express $V(r, R)$ as

$$
V(r, R)= \begin{cases}b^{-2}\left[1+c-2(1+b R) e^{-b R}(b r)^{-1} \sinh (b r)\right], & r<R \\ 2 b^{-2}[b R \cosh (b R)-\sinh (b R)](b r)^{-1} e^{-b r}-b^{-2}(1-c), & r>R .\end{cases}
$$

Hence we have

$$
Z(R)=b^{-2}\left\{1+c-\left[1+(b R)^{-1}\right]\left(1-e^{-2 b R}\right)\right\} \quad \text { for } R>0 .
$$

The graph of $Z(R)$ has a unique inflection point, say $\bar{R}$, and $Z(R)$ satisfies

$$
\begin{aligned}
& Z_{R R}(R)>0 \text { if } R \in(0, \bar{R}), \\
& Z_{R R}(\bar{R})=0, \\
& Z_{R R}(R)<0 \text { if } R \in(\bar{R}, \infty) .
\end{aligned}
$$

Thus the bifurcation curve of equilibrium balls is as in Fig. 2 in Sec. 1.

Next we study the stability. We will calculate $f(R)$ for $R>0$ with $Z(R)=U(R)$. Recall that

$$
L[w]=-\left(s^{2} w_{s}\right)_{s}+n(n+1)+s^{2}
$$

has linearly independent solutions $\sqrt{\pi /(2 z)} I_{n+\frac{1}{2}}(z)$ and $\sqrt{\pi /(2 z)} K_{n+\frac{1}{2}}(z)$, which are called modified Bessel functions. We find that $L[w]=h(s)$ is equivalent to $w(s)=$ $\int_{0}^{\infty} G_{n}(s, t) h(t) d t$. The Green function $G_{n}(s, t)$ is given by

$$
G_{n}(s, t)= \begin{cases}(s t)^{-\frac{1}{2}} K_{n+\frac{1}{2}}(t) I_{n+\frac{1}{2}}(s), & 0<s \leq t<+\infty \\ (s t)^{-\frac{1}{2}} I_{n+\frac{1}{2}}(t) K_{n+\frac{1}{2}}(s), & 0<t<s<+\infty .\end{cases}
$$

We have $Q(R) \equiv 2, g(r, R) \equiv b^{2}$, and

$$
\left(-\frac{d^{2}}{d r^{2}}-\frac{2}{r} \frac{d}{d r}+\frac{2}{r^{2}}+b^{2}\right) w_{1}(r, R)=\delta_{R}
$$

Putting $z_{1}(s)=w_{1}(r, R)$ with $s=b r$, we have

$$
\begin{aligned}
-\frac{d}{d s}\left(s^{2} \frac{d z_{1}}{d s}\right)+\left(2+s^{2}\right) z_{1}=0, \quad s \in(0, b R) \cup(b R, \infty), \\
-\left[\frac{d z_{1}}{d s}\right]_{b R-0}^{b R+0}=b^{-1} .
\end{aligned}
$$


This relation yields

$$
w_{1}(R, R)=z_{1}(b R)=R I_{\frac{3}{2}}(b R) K_{\frac{3}{2}}(b R)
$$

We have

$$
\left(-\frac{d^{2}}{d r^{2}}-\frac{2}{r} \frac{d}{d r}+\frac{6}{r^{2}}+b^{2}\right) w_{2}(r, R)=\delta_{R}(r)
$$

in $\left(X_{1}\right)^{\prime}$. Putting $z_{2}(s)=w_{2}(r, R)$ with $s=b r$, we have

$$
\begin{aligned}
-\frac{d}{d s}\left(s^{2} \frac{d z_{2}}{d s}\right)+\left(6+s^{2}\right) z_{2} & =0, \quad s \in(0, b R) \cup(b R, \infty), \\
& -\left[\frac{d z_{2}}{d s}\right]_{b R-0}^{b R+0}=b^{-1} .
\end{aligned}
$$

Using this relation and the Green function $G_{n}$, we obtain

$$
w_{2}(R, R)=z_{2}(b R)=R I_{\frac{5}{2}}(b R) K_{\frac{5}{2}}(b R) .
$$

Now we have

$$
p(R)=-W^{\prime}(V(R, R))=\frac{a_{2}}{\left(U(R)+a_{1}\right)^{2}}=\frac{4 \alpha^{2}}{a_{2} R^{2}} .
$$

From $Z(R)-U(R)=0$, we get

$$
\alpha=\frac{a_{2} R}{2\left\{b^{-2}(1+c)+a_{1}-b^{-2}\left[1+(b R)^{-1}\right]\left(1-e^{-2 b R}\right)\right\}} .
$$

Using the expression (5.3) of $f(R)$, we obtain

$$
\frac{1}{4 \alpha} f(R)=\frac{I_{\frac{3}{2}}(b R) K_{\frac{3}{2}}(b R)-I_{\frac{5}{2}}(b R) K_{\frac{5}{2}}(b R)}{b^{-2}(1+c)+a_{1}-b^{-2}\left[1+(b R)^{-1}\right]\left(1-e^{-2 b R}\right)}-\frac{1}{R^{2}} .
$$

We have

$$
\begin{aligned}
\sqrt{\frac{\pi}{2 z}} I_{\frac{3}{2}}(z) & =-z^{-2} \sinh z+z^{-1} \cosh z \\
\sqrt{\frac{\pi}{2 z}} K_{\frac{3}{2}}(z) & =\frac{\pi}{2} z^{-1} e^{-z}\left(1+z^{-1}\right) \\
\sqrt{\frac{\pi}{2 z}} I_{\frac{5}{2}}(z) & =\left(3 z^{-3}+z^{-1}\right) \sinh z-3 z^{-2} \cosh z \\
\sqrt{\frac{\pi}{2 z}} K_{\frac{5}{2}}(z) & =\frac{\pi}{2} z^{-1} e^{-z}\left(1+3 z^{-1}+3 z^{-2}\right)
\end{aligned}
$$

See [1] for instance. Thus we get

$$
I_{\frac{3}{2}}(z) K_{\frac{3}{2}}(z)-I_{\frac{5}{2}}(z) K_{\frac{5}{2}}(z)=\frac{1}{2 z^{5}}\left[\left(2 z^{2}-9\right)+\left(2 z^{4}+8 z^{3}+16 z^{2}+18 z+9\right) e^{-2 z}\right] .
$$

Substituting this relation into (6.7), we obtain

$$
f(R)=2 \alpha b^{2} F_{2}(b R) / F_{1}(b R)
$$




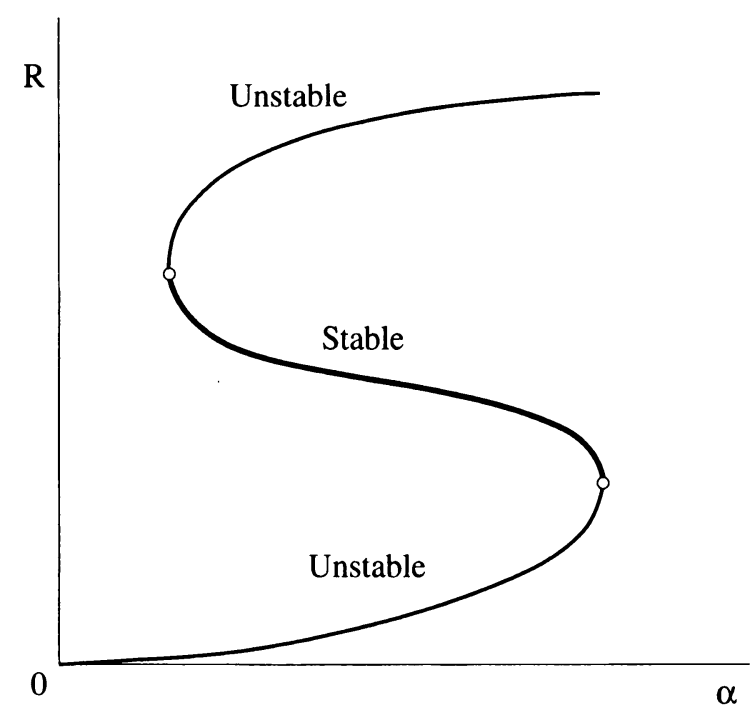

FIG. 4. Stability of equilibrium balls for Example 1

where

$$
\begin{array}{lll}
F_{1}(z) & \stackrel{\text { def }}{=} & z^{4}\left[\left(c+a_{1} b^{2}\right) z-1+(z+1) e^{-2 z}\right] \\
F_{2}(z) & \stackrel{\text { def }}{=} & -2\left(c+a_{1} b^{2}\right) z^{3}+4 z^{2}-9+\left(2 z^{4}+6 z^{3}+14 z^{2}+18 z+9\right) e^{-2 z}
\end{array}
$$

for $z>0$. The assumption $c+a_{1} b^{2}>1$ yields

$$
F_{1}(z)>0, \quad F_{2}(z)<0 \quad \text { for every } z>0 .
$$

Hence we have $f(R)<0$ for every $R>0$. If $R_{0}$ satisfies $Z\left(R_{0}\right)=U\left(R_{0}\right)$ and $\frac{d Z}{d R}\left(R_{0}\right)>$ $\frac{d U}{d R}\left(R_{0}\right)$, then an equilibrium ball $\left(\Gamma\left(R_{0}\right), V\left(r, R_{0}\right)\right)$ is stable. If $R_{0}$ satisfies $Z\left(R_{0}\right)=$ $U\left(R_{0}\right)$ and $\frac{d Z}{d R}\left(R_{0}\right)<\frac{d U}{d R}\left(R_{0}\right)$, then an equilibrium ball $\left(\Gamma\left(R_{0}\right), V\left(r, R_{0}\right)\right)$ is unstable. This implies that the stability of equilibrium balls is as drawn in Fig. 4.

6.2. Example 2. Consider

$$
\begin{aligned}
\tau V_{\Gamma(t)} & =-v-2 \alpha H \quad \text { on } \Gamma(t), t>0, \\
v_{t}-\Delta v & =\left(1-b^{2} v+c\right) \chi_{\Omega^{+}(t)}+\left(-1-b^{2} v+c\right) \chi_{\Omega^{-}(t)} \quad \text { in } \mathbf{R}^{3}, t>0 .
\end{aligned}
$$

Here $b>0$ and $0<c<1$ are positive constants. The associated reaction-diffusion equation is (1.6) with $W(v)=-v$. Functions $V(r, R)$ and $Z(R)$ are as in (6.3) and (6.4). We have $Q(R) \equiv 2, p(R) \equiv 1$, and $U(R)=-2 \alpha / R$. The graphs of $Z(R)$ and $U(R)$ have at most two intersection points. Let $R>0$ satisfy $Z(R)=U(R)$. Then $Z(R)=U(R)$ yields

$$
\alpha=-\frac{1}{2} b^{-2} R\left\{1+c-\left[1+(b R)^{-1}\right]\left(1-e^{-2 b R}\right)\right\} .
$$

Functions $w_{1}(r, R)$ and $w_{2}(r, R)$ are given by (6.5) and (6.6). Using (5.3), we obtain

$$
f(R)=2 R\left[I_{\frac{3}{2}}(b R) K_{\frac{3}{2}}(b R)-I_{\frac{5}{2}}(b R) K_{\frac{5}{2}}(b R)\right]-\frac{4 \alpha}{R^{2}} .
$$




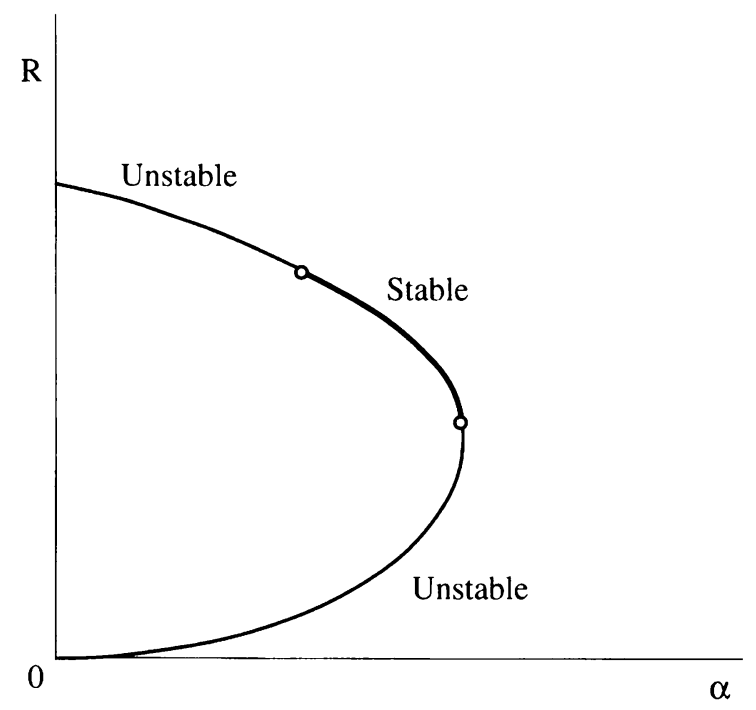

FIG. 5. Stability of equilibrium balls for Example 2

Using (6.8), we have, after simple calculation,

$$
f(R)=R F_{4}(b R) / F_{3}(b R),
$$

where

$$
\begin{aligned}
& F_{3}(z)=z^{5} e^{2 z}>0 \\
& F_{4}(z)=\left(2 c z^{3}-9\right) e^{2 z}+2 z^{4}+10 z^{3}+18 z^{2}+18 z+9
\end{aligned}
$$

for $z>0$. After elementary computation, we find that $F_{4}(\cdot)$ has a unique zero point $z_{0}$ with $F_{4}\left(z_{0}\right)=0$,

$$
\begin{array}{ll}
F_{4}(z)<0 & \text { for } z \in\left(0, z_{0}\right), \\
F_{4}(z)>0 & \text { for } z \in\left(z_{0}, \infty\right) .
\end{array}
$$

Thus, if $R_{0}$ satisfies $Z\left(R_{0}\right)=U\left(R_{0}\right)$ and $\frac{d Z}{d R}\left(R_{0}\right)>\frac{d U}{d R}\left(R_{0}\right)$, then $\left(\Gamma\left(R_{0}\right), V\left(r, R_{0}\right)\right)$ is stable if $R<b^{-1} z_{0}$ and is unstable if $R>b^{-1} z_{0}$.

Consider the case where $\alpha \rightarrow+0$. It is shown in [4] that (5.5) gives $\widehat{\kappa}=\left(4 \alpha / R_{0}^{*}\right)^{-2 / 3}$ $+O(1)$ as $\alpha \rightarrow+0$ and the associated eigenvalue satisfies $\lim _{\alpha \rightarrow 0} \Lambda\left(\widehat{\kappa}, R_{0}\right)=$ $-\tau^{-1} p\left(R_{0}^{*}\right) V_{r}\left(R_{0}^{*}, R_{0}^{*}\right)>0$. Here $R_{0}^{*}>0$ is a unique $R=R_{0}^{*}$ that satisfies $Z\left(R_{0}^{*}\right)=0$. This implies that $\left(\Gamma\left(R_{0}\right), V\left(r, R_{0}\right)\right)$ is unstable if $\left|R_{0}-R_{0}^{*}\right|$ is small enough. Combining this fact and Theorem 2, the stability of $\left(\Gamma\left(R_{0}\right), V\left(r, R_{0}\right)\right)$ turns out to be as in Fig. 5 .

In the case of $\alpha \rightarrow+0$, the associated eigenfunction $\rho(\xi)$ consists of spherically harmonic functions of large degrees, which are given by $\left(4 \alpha / R_{0}^{*}\right)^{-1 / 3}+O(1)$ as $\alpha \rightarrow+0$. This implies that instability of an equilibrium ball happens with high modes if it is located far from the bifurcation points in the bifurcation diagram.

7. Acknowledgments. The author expresses his sincere gratitude to Prof. Xinfu Chen for his advice and suggestions. Special thanks go to Prof. Tohru Tsujikawa for 
explaining his recent work [9], which inspired the author to obtain Lemma 6. He also thanks Dr. Hirokazu Ninomiya for his comments on Sec. 6.

\section{REFERENCES}

[1] M. Abramowitz and I. A. Stegun, Handbook of Mathematical Functions, Dover Publications, New York, 1964

[2] X. Chen, Generation and propagation of interfaces in reaction-diffusion systems, Trans. Amer. Math. Soc. (2) 334, 877-913 (1992)

[3] X-Y. Chen, Dynamics of interfaces in reaction diffusion systems, Hiroshima Math. J. 21, 47-83 (1991)

[4] X. Chen and M. Taniguchi, Instability of spherical interfaces in a nonlinear free boundary problem, to appear in Advances in Differential Equations

[5] P. C. Fife, Dynamics of internal layers and diffusive interfaces, CBMS-NSF Regional Conference Series in Applied Mathematics 53, SIAM, Philadelphia, 1988

[6] C. Müller, Spherical Harmonics, Lecture Notes in Mathematics 17, Springer-Verlag, Berlin, Heidelberg, New York, 1966

[7] Y. Giga, S. Goto, and H. Ishii, Global existence of weak solutions for interface equations coupled with diffusion equations, SIAM J. Math. Anal. 23, 821-835 (1992)

[8] D. Hilhorst, Y. Nishiura, and M. Mimura, A free boundary problem arising in some reactiondiffusion systems, Proc. Roy. Soc. Edinburgh 118A, 355-378 (1991)

[9] M. Mimura and T. Tsujikawa, Aggregating pattern dynamics in a chemotaxis model including growth, Physica A 230, 499-543 (1996)

[10] Y. Nishiura and M. Mimura, Layer oscillations in reaction-diffusion systems, SIAM J. Appl. Math. 49, 481-514 (1989)

[11] T. Ohta, M. Mimura, and R. Kobayashi, Higher-dimensional localized patterns in excitable media, Physica D 34, 115-144 (1989)

[12] M. Taniguchi and Y. Nishiura, Instability of planar interfaces in reaction-diffusion systems, SIAM J. Math. Anal. (1) 25, 99-134 (1994) 